\title{
A Combinatória em Livros Didáticos do Ensino Fundamental
}

\author{
Monalisa Cardoso Silva \\ Cristiane Azevêdo dos Santos Pessoa
}

\begin{abstract}
Resumo: O presente estudo buscou analisar o trabalho com a Combinatória em livros didáticos de Matemática do Ensino Fundamental, à luz da Teoria dos Campos Conceituais e de algumas pesquisas sobre o assunto. Foi selecionada uma coleção de cada nível do Ensino Fundamental - anos iniciais e anos finais - entre as aprovadas pelo PNLD 2013 e 2014, respectivamente. Por meio do mapeamento de todas as atividades e do manual do professor, foi possível perceber que, embora os problemas combinatórios estejam presentes e ocorra uma boa variedade de representações simbólicas, é necessário explorar a percepção das propriedades invariantes envolvidas na Combinatória e as orientações específicas para que o docente, principalmente os dos anos iniciais, para os quais, de um modo geral, não existe uma formação aprofundada sobre esse conhecimento, trabalhe tal conhecimento de forma a propiciar ao aluno a construção e o desenvolvimento pleno desse conteúdo.
\end{abstract}

Palavras-chave: Livros didáticos; Combinatória; Ensino Fundamental

\section{The Combinatorial in Textbooks of Elementary School}

Abstract: This study sought to analyze the development of the subject Combinatorics in Math Textbooks of Elementary School in the light of the Theory of Conceptual Fields and some research that approach this topic. A collection if each level of Elementary School was selected - early years and final years - between those that were approved by PNLD 2013 and following year, respectively. Through the mapping of all activities and the Teacher's Guide, was possible to realize that, although the combinatorial problems are present and there is a good variety of symbolic representations, it's necessary to explore the perception of the invariant properties that are involved in Combinatorics and to formulate specific guidelines that makes the teacher capable to develop this subject effectively with the student, mainly the teacher of the early years, who generally, don't have in-depth training about this specific knowledge.

Keywords: Textbooks; Combinatorics; Elementary School

\section{Introdução}

O raciocínio combinatório é uma forma de pensar que permite que se levantem possibilidades e sejam analisadas as suas combinações, auxiliando na compreensão de

1 Mestra em Educação Matemática e Tecnológica pela Universidade Federal de Pernambuco (UFPE), câmpus de Recife. Email: monalisacardosoo8@yahoo.com.br.

2 Doutora em Educação pela Universidade Federal de Pernambuco (UFPE), Professora e pesquisadora do Edumatec/UFPE - câmpus de Recife. Email: cristianepessoa74@gmail.com. 
conteúdos matemáticos e de outras áreas do conhecimento (Pessoa \& Borba, 2010). Portanto, é um tipo de pensamento que deve ser valorizado pela escola desde cedo, pois pode auxiliar os alunos no desenvolvimento do raciocínio lógico, assim como na resolução de diversos tipos de problemas relacionados ou não à Matemática. Segundo Pessoa (2014), ele desenvolve o pensamento hipotético-dedutivo, estimulando os alunos a pensar no que é possível e, não necessariamente, no que é real.

Os Parâmetros Curriculares Nacionais - PCN (MEC, 1997), sobre esse conhecimento, recomendam para o $1^{\circ}$ e $2^{\circ}$ ciclos "levar o aluno a lidar com situações-problema que envolvam combinações, arranjos e permutações e, principalmente, o princípio fundamental da contagem” (p. 57) e, para o 3o e 4o ciclos, referindo-se aos problemas de contagem, dizem que "o objetivo é levar o aluno a lidar com situações que envolvam diferentes tipos de agrupamentos que possibilitem o desenvolvimento do raciocínio combinatório e a compreensão do princípio multiplicativo para aplicação no cálculo de probabilidade” (MEC, 1998, p.52).

No que concerne às orientações para o trabalho com a Matemática no Ensino Médio, é indicada a Combinatória, porque ela tem um papel importante para o desenvolvimento do raciocínio lógico, uma vez que ele contribui para a resolução de problemas no dia a dia. Por outro lado, a Combinatória é um dos suportes para a aprendizagem de outro conteúdo importante na área da Matemática: a Probabilidade.

Acreditamos, assim como Borba (2013), que haverá possibilidade de um mais amplo desenvolvimento do raciocínio combinatório se ocorrer, desde os anos iniciais do Ensino Fundamental, o trabalho com os variados problemas deste campo conceitual, por meio de representações simbólicas apropriadas que possibilitem uma gradual construção de procedimentos mais formais e da compreensão das propriedades invariantes do conceito existente, até se chegar ao uso consciente das fórmulas de Análise Combinatória no Ensino Médio.

Apesar das orientações encontradas nos PCN, de acordo com Pessoa e Borba (2009), na prática de ensino de sala de aula, a maioria dos problemas combinatórios (arranjo, combinação e permutação) é introduzida, formalmente, tão somente no 2o ano do Ensino Médio. O que se observa na realidade das aulas dos primeiros ciclos da Educação Básica é o trabalho apenas com o problema do tipo produto cartesiano de forma explícita e sistemática.

O adiamento do ensino sistematizado de problemas que envolvam o raciocínio combinatório desde os anos iniciais pode refletir nas dificuldades demonstradas por alunos nos anos finais da escolarização básica. Ou seja, pode ocorrer que os alunos se transformem em meros aplicadores de fórmulas, uma vez que o trabalho com esse conhecimento é feito em um curto período de tempo, de forma não sistematizada e, algumas vezes, até insegura no que se refere ao conhecimento do conteúdo por parte dos professores. 
Dessa forma, baseadas em Barreto, Amaral e Borba (2007) e em Assis e Magalhães (2012) e diferenciando-nos por incluir no presente estudo os anos finais do Ensino Fundamental, buscamos analisar o desenvolvimento da Combinatória em livros didáticos de Matemática do Ensino Fundamental. Para tal, apoiamo-nos na Teoria dos Campos Conceituais, proposta por Vergnaud (1986), que apresenta a formação de um conceito a partir de três pilares: situações, invariantes e representações simbólicas.

\section{A Combinatória e a Teoria dos Campos Conceituais}

Merayo (2001) define a Análise Combinatória como a técnica de saber quantos objetos há em um conjunto sem ter que contá-los um a um. Dessa maneira, a contagem na Análise Combinatória não é vista unicamente como enumeração direta de elementos, mas como determinação de possibilidades, sem necessariamente levantar todos os casos possíveis.

Rosa (2004) entende a Combinatória como o campo da Matemática que permite resolver situações nas quais é necessário "escolher e organizar" objetos de um conjunto, ou seja, é a parte da Matemática na qual estudamos formas de contagem de agrupamentos de um conjunto ou mais.

Sendo assim, a Combinatória também é conhecida como a arte de contar, a qual é uma das primeiras aprendizagens matemáticas do sujeito, quando a criança conta ou enumera elementos (Morgado, Pitombeira de Carvalho, Carvalho, \& Fernandez, 1991). Esse conhecimento tem origem em jogos de azar, com cartas e lançamentos de dados, e vem se desenvolvendo e apresentando aplicabilidade em diversas áreas, como "no cálculo das probabilidades, em problemas de transporte, na confecção de horários, de elaboração de planos de produção, de programação linear, de estatística, de teoria da informação, de biologia molecular, de economia, de logística, etc" (Pessoa, 2009, p. 72).

Para embasar o presente estudo, apoiamo-nos na classificação dos problemas combinatórios feita em uma organização por Pessoa e Borba (2009), que se basearam na Teoria dos Campos Conceituais de Vergnaud (1986). Para esse autor, "um campo conceitual pode ser definido como um conjunto de situações, cujo domínio requer uma variedade de conceitos, de procedimentos e de representações simbólicas em estreita conexão" (p. 84). Para compreender um campo conceitual, é necessária a análise das situações envolvidas, os procedimentos de resolução utilizados pelos alunos, suas argumentações e as representações simbólicas utilizadas.

Vergnaud (1986) defende que todo conceito é constituído por três dimensões: as situações que dão significado ao conceito, os invariantes (relações e propriedades) 
que se mantêm constantes nas diversas situações e as representações utilizadas para simbolizar o conceito.

Desta forma, Pessoa e Borba. (2009) classificam os problemas que envolvem raciocínio combinatório, assumindo os seguintes significados: produto cartesiano, combinação, arranjo e permutação.

Analisando a Combinatória com base no tripé estabelecido por Vergnaud, os significados se referem aos tipos de problemas envolvidos nesse raciocínio (arranjo, combinação, permutação e produto cartesiano). Acreditamos assim, que esses são uma variável importante no processo de ensino, na compreensão e na aplicação de um conceito, pois, em cada um dos problemas combinatórios, o aluno se utilizará de relações lógicas diferentes.

Os diferentes invariantes do conceito são elementos fundamentais para que se compreendam as lógicas implícitas em cada significado da Combinatória, interferindo, assim, na forma de os alunos compreenderem cada tipo de problema. Nas situações combinatórias, esses invariantes dizem respeito à escolha dos elementos que formarão os subconjuntos, se serão utilizados todos ou parte deles em cada possibilidade; e à ordem em que os elementos estarão dispostos nessas possibilidades, gerando ou não uma nova possibilidade.

Os problemas podem ser resolvidos por meio de diferentes formas de representação: desenhos, listagens, árvores de possibilidades, tabelas, fórmulas, dentre outras, como constatado em estudos de sondagens como o de Pessoa e Borba, (2009). As diferentes formas de representação simbólica ocorrem no que se refere tanto às soluções apresentadas pelos alunos quanto aos enunciados das questões propostas.

\section{O livro didático e sua importância para o ensino}

O livro didático é um dos recursos mais utilizados no processo de ensino e aprendizagem, sendo, assim, um dos mais importantes componentes do cotidiano das escolas em todos os níveis de ensino, desempenhando um papel de extrema relevância no sistema escolar. É principalmente com base nesse instrumento que o professor norteia o seu trabalho, muitas vezes o único instrumento pedagógico de que dispõe.

Dante (1996) aborda o livro didático como orientador do trabalho do professor em sala de aula no que diz respeito a objetivos a serem alcançados, conteúdos principais a serem desenvolvidos, metodologias e estratégias a serem utilizadas para alcançar tais objetivos. Entretanto, o livro didático precisa ser visto como um recurso auxiliar no processo de ensino e aprendizagem e, não, como dominante nesse processo.

Com o objetivo principal de auxiliar o trabalho pedagógico dos professores por meio da distribuição de coleções de livros didáticos aos alunos da Educação Básica, o Ministério da Educação (MEC) publica, a partir do ano de 1996, o Guia de Livros 
Didáticos (MEC, 1996) com resenhas das coleções aprovadas. Nele, as coleções são analisadas, baseando-se em critérios estabelecidos que propiciem as adequações de aspectos teórico-metodológicos, estrutura editorial e manual do professor. O guia é encaminhado às escolas, que escolhem, entre as coleções disponíveis, aquelas que melhor atendem ao seu projeto político pedagógico.

Tendo o livro didático como um instrumento referencial com relação ao saber a ser ensinado e um condutor dos conteúdos e dos processos envolvidos na prática pedagógica do professor, faz-se necessário analisar como a Combinatória está organizada e estruturada nos livros didáticos destinados às escolas brasileiras.

\section{O que pesquisas apontam sobre o livro didático e a Combinatória}

Barreto, Amaral e Borba (2007) analisaram como livros didáticos de Matemática do Ensino Fundamental, aprovados pelo Programa Nacional do Livro Didático (PNLD) 2007, abordavam o raciocínio combinatório. Foi realizada uma seleção aleatória de cinco coleções dentre as aprovadas pelo PNLD e a análise foi feita à luz da Teoria dos Campos Conceituais de Vergnaud. Nesse estudo foi constatado que os significados da Combinatória se fazem presentes nas coleções de livros dos anos iniciais do Ensino Fundamental, sendo os mais presentes, os de combinação e produto cartesiano; que os livros os apresentam em seus enunciados e sugerem uma diversidade de representações simbólicas para resolução, mas não trazem nenhuma orientação ao professor no que diz respeito aos significados combinatórios e às características invariantes deles.

Assis e Magalhães (2012) buscaram fazer um estudo comparativo com o citado anteriormente, analisando, dessa vez, coleções aprovadas pelo PNLD 2010. As coleções selecionadas foram as mesmas do estudo em comparação. Foi constatado que, confrontando os livros do PNLD de 2007 e os de 2010, houve uma diminuição na quantidade total dos problemas que envolvem raciocínio combinatório, e, dentre as representações simbólicas abordadas, se destacaram os desenhos e os enunciados apenas em linguagem natural. Contudo, não houve diferença significativa entre as representações simbólicas nos dois estudos.

Oliveira e Coutinho (2013) discutiram como o conteúdo de Combinatória é abordado nos livros didáticos de anos iniciais do Ensino Fundamental. Para tal, foi feita uma análise de como o tema é abordado nos livros de Alfabetização Matemática e Matemática, a partir dos sumários e das resenhas de cada coleção aprovada pelo PNLD 2013. As autoras também tomaram como base de análise orientações presentes nos PCN e alguns resultados de pesquisas na área de Educação Matemática, juntamente com pressupostos da Teoria da Transposição Didática. As análises 
realizadas permitiram às autoras inferir que o tratamento dado pelos autores aos conteúdos desde campo não é sequencial, o que dificulta o aprofundamento do conhecimento dos alunos. Observaram ainda que, na maior parte das coleções, ele não é abordado no bloco de Tratamento da Informação, mas, sim, como aplicação do princípio multiplicativo.

Podemos inferir, por meio dos resultados dos estudos citados, que a abordagem da Combinatória nos livros didáticos ainda precisa evoluir no que diz respeito às necessidades de aprendizagem do aluno e ao ensino do professor. Esse campo de estudo merece atenção e necessidade de investigação ao longo de toda a Educação Básica, uma vez que seu ensino ganha êxito, apenas, no final do Ensino Médio.

\section{Método}

Com o objetivo de analisar o desenvolvimento da Combinatória em livros didáticos de Matemática do Ensino Fundamental (anos iniciais e anos finais) à luz da Teoria dos Campos Conceituais, foi selecionada uma coleção de cada nível do Ensino Fundamental entre as aprovadas pelo PNLD 2013 (anos iniciais) e 2014 (anos finais), de forma aleatória, ou seja, dentre o universo de livros aprovados, escolhemos uma coleção de cada nível de ensino, às quais tínhamos acesso.-

Foram analisados todos os volumes das coleções, tanto no livro do aluno quanto no manual do professor, sendo feito o levantamento das atividades, incluindo exemplos, exercícios a resolver, exercícios resolvidos e explicações teóricas, dentro e fora dos capítulos e/ou secções destinados à Combinatória, e conteúdos e temas a ela relacionados. Havendo uma atividade com questões $a, b$ e $c$, cada item foi contabilizado e tomado separadamente para análise.

Como base teórica da pesquisa, adotamos a Teoria dos Campos Conceituais (Vergnaud, 1986), sendo feita a identificação das situações combinatórias e representações simbólicas apresentadas e sugeridas nas atividades dos livros, assim como a análise da compreensão das propriedades invariantes do conceito nas atividades mapeadas. Também analisamos a ocorrência de mudança/gradação de dificuldades na abordagem dos problemas combinatórios ao longo dos anos escolares e se o manual do professor orienta o docente para o trabalho com a Combinatória.

\section{Resultados e análise dos dados}

\section{Anos iniciais do Ensino Fundamental}

Pelo mapeamento das atividades relacionadas ao raciocínio combinatório, na coleção em questão, foi possível encontrar um quantitativo total de 39 problemas ao longo dos cinco livros da coleção. Há apenas problemas a resolver e um jogo, 
não existindo assim, exemplos, exercícios respondidos e explicações. No Gráfico 1, a seguir, observamos como tais problemas estão distribuídos por ano de escolarização:

Gráfico 1. Quantitativo de problemas combinatórios distribuídos em cada ano escolar

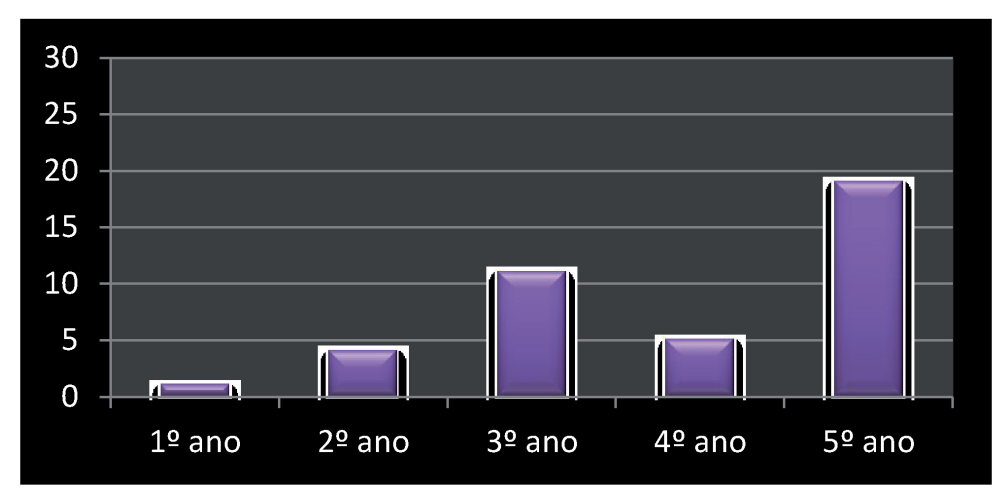

Os referidos problemas combinatórios não se integram num capítulo ou numa secção específica que os agrupe. No geral, estão contemplados em atividades que envolvem estruturas multiplicativas e aditivas, conforme visto por Oliveira e Coutinho. (2013) nas resenhas dos livros. Percebemos que eles começam a aparecer em quantidade mais expressiva a partir do final do ciclo de alfabetização matemática (3ano), mas no 4 ano a quantidade cai e volta a subir bastante no 5o ano, ou seja, não há um crescimento sistemático no número de problemas, à medida que os anos escolares avançam.

No que diz respeito aos significados (tipos) de problemas combinatórios, eles estão distribuídos, como descrito no Quadro 1, apresentado a seguir.

Quadro 1: Frequência de problemas por significados em cada ano escolar

\begin{tabular}{|c|c|c|c|c|}
\hline \multirow[t]{2}{*}{$\begin{array}{l}\text { Ano } \\
\text { escolar }\end{array}$} & \multicolumn{4}{|c|}{$\begin{array}{l}\text { Quantidade de problemas combinatórios por } \\
\text { significado }\end{array}$} \\
\hline & $\begin{array}{l}\text { Produto } \\
\text { Cartesiano }\end{array}$ & Combinação & Arranjo & Permutação \\
\hline $1^{\circ}$ ano & 0 & 1 & 0 & 0 \\
\hline $2^{\circ}$ ano & 0 & 3 & 1 & 0 \\
\hline $3^{\circ}$ ano & 4 & 2 & 0 & 5 \\
\hline $4^{\circ}$ ano & 3 & 1 & 0 & 1 \\
\hline $5^{\circ}$ ano & 13 & 1 & 0 & 3 \\
\hline Total & 20 & 8 & 1 & 9 \\
\hline
\end{tabular}

Notamos, nessa coleção, que todos os significados da Combinatória se fazem presentes, entretanto a diversidade de tipos de problemas não é contemplada em cada livro. Nos primeiros anos da alfabetização matemática, eles quase não aparecem, há só o tipo combinação. Nos anos seguintes, existe uma predominância dos problemas de produto cartesiano, seguida dos de permutação e combinação. Os 
do tipo arranjo só aparecem uma única vez no livro do 2o ano. Os do tipo produto cartesiano são os mais frequentes e aumentam do 3/4 anos para o 5‥ Esse é o tipo comum de problema multiplicativo e é, nesses anos de escolarização, que esse conteúdo é introduzido e aprofundado. Os demais tipos de problema, como visto no estudo de Barreto, Amaral e Borba (2007) e no de Assis e Magalhães (2012), têm menor frequência. Esses tipos são mais explorados no 2o ano do Ensino Médio, conforme mostrou o estudo de Silva (2014).

O resultado observado é contrário ao que Vergnaud (1986) defende para a compreensão de conceitos. Para ele é necessário que se saiba que uma situação não representa todas as propriedades de um conceito, ou seja, para que o aluno compreenda todas as propriedades da Combinatória, é preciso que os livros façam uso da diversidade de classes de problemas que esse conceito envolve.

Uma das questões do 5a ano não diz respeito a um problema específico, antes se faz uma reflexão sobre a semelhança entre um conjunto destes tipos de problemas para que o aluno pense sobre a diferença entre os problemas combinatórios e outros problemas multiplicativos. O livro apresenta problemas envolvendo a ideia de combinar elementos e, por fim, na questão seguinte, solicita que o aluno reflita sobre o que notou de comum entre os problemas, pretendendo que ele perceba que a multiplicação também envolve as possibilidades de combinar elementos, como mostra a Figura 1.

Figura 1: Atividade sobre a semelhança entre um conjunto de problemas combinatórios

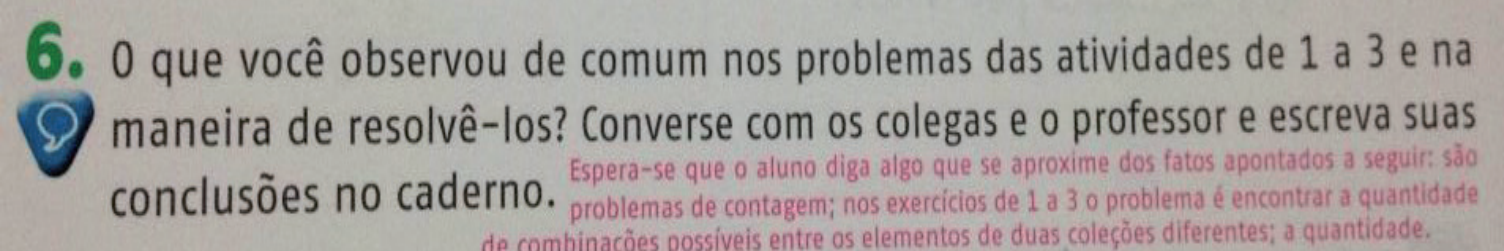

FONTE: Projeto Prosa. Daniela Padovan, Isabel C. Guerra, Ivonildes Milan e Priscila Monteiro. Editora Saraiva, 2011, 5o ano, p. 227.

Esse bloco de atividades é apresentado após o trabalho com uma sequência de problemas de estrutura multiplicativa. Assim, acreditamos que existe uma busca, na atividade 6 apresentada na Figura 1, acima, de, ao trabalhar cada uma das ideias da multiplicação, levar o aluno a perceber a diferença de raciocínio matemático envolvendo aspectos do pensamento combinatório, e, por último, com a ideia combinatória levantar tais questionamentos ao aluno. Esse aspecto se faz importante uma vez que, apesar de os problemas combinatórios estarem inseridos no campo das estruturas multiplicativas, são de problemas com maior complexidade e não tão comuns como os demais, pois nem sempre são resolvidos por meio de uma multipli- 
cação direta, estratégia de resolução típica dos problemas multiplicativos. Trata-se de um tipo de contagem que exige a superação da simples ideia de enumeração de elementos de um conjunto para passar à contagem de grupos de objetos, ou seja, de subconjuntos, tendo como base o raciocínio multiplicativo (Morgado, Pitombeira de Carvalho, Carvalho, \& Fernandez, 1991).

De modo geral, podemos inferir, assim, que, embora apareçam nos livros dos anos iniciais, da coleção analisada, todos os tipos de problemas, eles não são trabalhados em sua totalidade, no que diz respeito aos seus significados. Em cada ano escolar, há uma predominância de alguns, e outros são praticamente esquecidos, o que pode dificultar o contato do aluno com todas as situações para que possa perceber seus invariantes e especificidades.

Analisando as formas de representação simbólica utilizadas para apresentar as atividades, notamos que, em sua maioria, os livros da coleção apresentam os problemas com apenas o enunciado verbal $(48,7 \%)$ ou com um desenho $(38,4 \%)$ junto com o enunciado. Esse desenho, em geral, consiste numa figura relacionada ao contexto da atividade, servindo, muitas vezes, de apoio ao pensamento do aluno e auxiliando-o na resolução das questões. Algumas vezes esse desenho vem também seguido de uma tabela/quadro $(5,1 \%)$.

A maneira como o problema é apresentado pode gerar diferentes formas de resolução e de representação pelos alunos. Nunes, Campos, Magina e Bryant (2001) relacionam a forma de apresentação dos problemas com o nível de sucesso dos alunos. Há mais êxito quando a apresentação ocorre de maneira prática. Entretanto, esses autores defendem também a necessidade de trabalhar com problemas apresentados de forma esquemática, com desenhos, por exemplo, como no caso do livro didático.

No que diz respeito às representações simbólicas (estratégias) sugeridas para a resolução de cada problema, no geral a coleção apresenta uma diversidade de sugestões, como listagens, desenhos e quadros, como podemos ver no Quadro 2:

Quadro 2: Frequência de representações simbólicas sugeridas nos problemas em cada ano escolar

\begin{tabular}{|c|c|c|c|c|c|c|}
\hline Ano & \multicolumn{6}{|c|}{ Representações simbólicas sugeridas } \\
\cline { 2 - 7 } escolar & Listagem & Desenho & Quadro & Conta & Várias & Nenhuma \\
\hline $\mathbf{1}^{\mathbf{0}}$ ano & 1 & 0 & 0 & 0 & 0 & 0 \\
\hline $\mathbf{2}^{\mathbf{0}}$ ano & 0 & 1 & 0 & 0 & 0 & 3 \\
\hline $\mathbf{3}^{\mathbf{0}}$ ano & 0 & 1 & 1 & 0 & 2 & 7 \\
\hline $\mathbf{4}^{\mathbf{0}}$ ano & 1 & 0 & 0 & 0 & 0 & 4 \\
\hline $\mathbf{5}^{\mathbf{0}}$ ano & 0 & 0 & 1 & 7 & 6 & 4 \\
\hline Total & $\mathbf{2}$ & $\mathbf{2}$ & $\mathbf{2}$ & $\mathbf{7}$ & $\mathbf{8}$ & $\mathbf{1 8}$ \\
\hline
\end{tabular}

Obs. Várias significa: “questões que sugeriam mais de uma forma de resolução”. 
Apesar de serem apresentadas diferentes sugestões de resolução ao longo da coleção, que propõe algumas estratégias para resolver as questões, existe um alto quantitativo de problemas que apresentam apenas o enunciado. Esse aspecto é também identificado em pesquisas anteriores, como em Barreto, Amaral e Borba (2007) e em Assis e Magalhães (2012). Comparando esses estudos e o atual, observamos uma diminuição no uso do desenho como sugestão de resolução, estratégia essa bastante utilizada e com sucesso por alunos deste ciclo de escolarização, devendo, portanto, ser mais explorada.

Como já foi visto, dentre as estratégias sugeridas pela coleção nos enunciados dos problemas, verificamos um quantitativo alto de questões que não sugerem ao aluno qualquer forma de resolução. Entretanto, em alguns problemas é sugerido que o aluno resolva por mais de uma estratégia ou escolha uma que acredite ser mais viável, como mostra a Figura 2, a seguir.

Figura 2: Problema de produto cartesiano

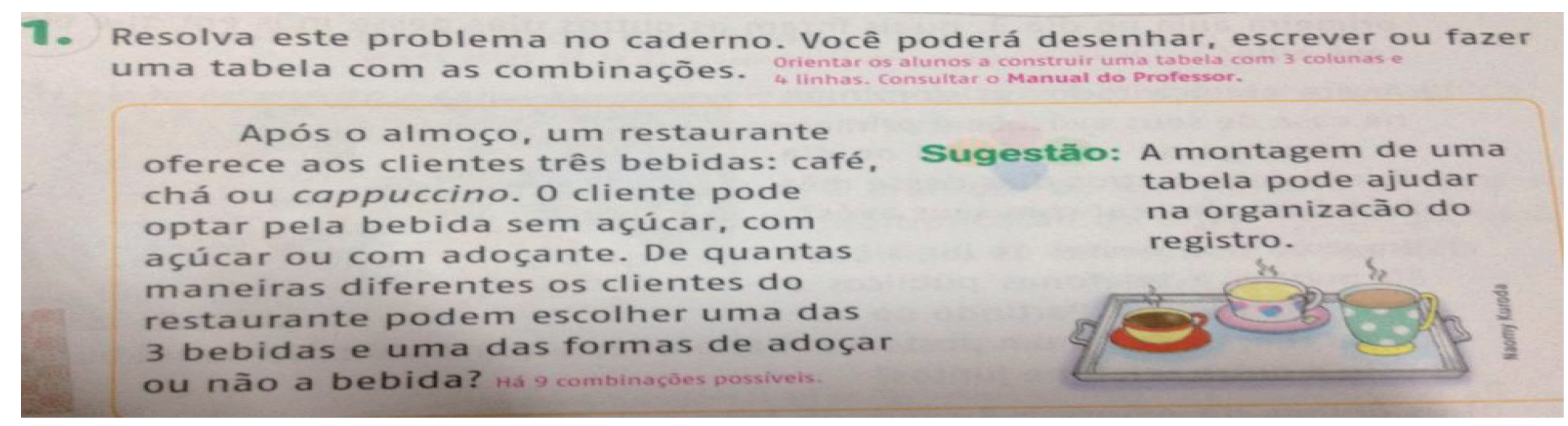

FONTE: Projeto Prosa. Daniela Padovan, Isabel C. Guerra, Ivonildes Milan e Priscila Monteiro. São Paulo: Saraiva, 2011, 5o ano, p. 227.

Esse aspecto é fundamental e enfatizado na perspectiva estabelecida em um dos pilares do tripé de Vergnaud (1986). O contato e o incentivo ao uso diversificado de representações simbólicas levarão os alunos a pensar sobre um determinado conceito a partir de diferentes perspectivas, e escolher a forma que lhe pareça mais apropriada para a resolução da situação dada.

Algumas vezes também as questões retomam problemas anteriores, solicitando que o aluno pense em uma operação que represente a sua resolução, antes resolvida por uma estratégia não formal. Desse modo, o aluno tem a possibilidade de refletir sobre o problema, por meio de diferentes representações, até chegar ao seu registro formal, pelo cálculo.

No que diz respeito à explicitação dos invariantes de cada tipo de problema combinatório (a ordem e a escolha dos elementos para a formação dos subconjuntos), elemento importante para que se compreendam as lógicas implícitas em cada significado, a coleção, nos enunciados das questões, não faz nenhuma menção a essas 
particularidades, tão pouco faz com que o aluno compare problemas combinatórios distintos e assim perceba tais invariantes entre eles.

Pelo mapeamento das atividades, foi possível verificar que não há uma gradação de dificuldade entre os anos escolares, mas tão somente um aumento na quantidade de problemas combinatórios abordados. Dessa forma, a partir da coleção analisada, podemos inferir que não existe uma evolução da abordagem da Combinatória do 1ํㅡㄴ ao 5a ano do Ensino Fundamental, sendo trabalhados problemas do mesmo grau de complexidade ao longo dos anos escolares desse ciclo.

Os contextos dos problemas apresentados pela coleção são adequados à faixa etária dos alunos, entretanto se repetem ao longo dos livros para cada tipo de problema. Em geral, os de produto cartesiano dizem respeito a possibilidades de conjuntos de roupas e de comidas e pares de pessoas; os de combinação, a possibilidades de dois algarismos que somados formam um determinado número; os de arranjo, a jogadas de dados; e os de permutação, à formação de números e cores de bandeiras.

As variáveis tomam, na maioria das vezes, valores numéricos baixos para as quantidades de possibilidade, para que o aluno não se perca nos cálculos, pois o propósito principal, nesse ciclo de escolarização, é a compreensão do problema e não a sentença numérica.

Analisando o manual do professor dessa coleção, assim como percebido em Barreto et al. (2007) e em Assis e Magalhães. (2012), não existe uma orientação específica ao ensino dos problemas combinatórios. Em alguns livros da coleção, são encontradas algumas sugestões de estratégias de como o aluno pode resolver os problemas, assim como, ao longo das atividades, há observações que só aparecem no livro do professor.

No manual não existem orientações ao professor para que ele possa explicitar aos alunos as diferenças dos invariantes do conceito de Combinatória, mas apenas que eles sejam levados a perceber as diferenças deste tipo de problema dos outros multiplicativos. As orientações específicas para o trabalho com a Combinatória se fazem importantes, pois além da iniciação à aprendizagem e ao desenvolvimento desse conhecimento, as crianças precisam também superar obstáculos possíveis nesse processo, tais como a não contagem de todas as possibilidades, uma vez que o trabalho com a Combinatória exige uma organização dos dados de forma particular. Segundo Pessoa (2014), essa organização é realizada em níveis diferenciados de abstração, que culmina em fórmulas nos anos finais do Ensino Médio. Sendo assim, orientações ao professor, tais como auxiliar as crianças na sistematização de suas estratégias e no desenvolvimento de ferramentas, podem ser úteis para a compreensão da Combinatória. 


\section{Anos finais do Ensino Fundamental}

Realizando o mapeamento das atividades de Combinatória nos livros didáticos da coleção, foi possível encontrar um quantitativo de 54 problemas envolvendo este conceito. Visualizamos a distribuição de tais questões ao longo dos anos escolares deste ciclo no Gráfico 2, a seguir.

Gráfico 2. Quantitativo de problemas combinatórios distribuídos em cada ano escolar

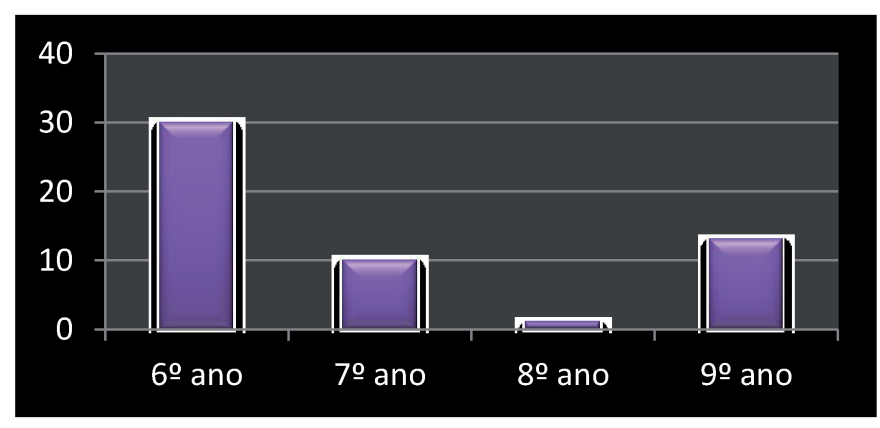

Em todos os livros dessa coleção são encontrados problemas de Combinatória, com mais ênfase no 6o ano, seguido do 9o e 7o anos. No livro do 8o ano, há apenas um problema combinatório, que está em uma ficha de revisão cumulativa e testes, com problemas retirados de avaliações externas e do tipo de escolha múltipla. As questões encontradas, em sua maioria, são problemas a resolver. Dentre elas, um exemplo, e três delas apresentam explicações (uma no 6oㅡ, uma no 7o e uma no 9o ano) sobre os problemas, como mostra a Figura 3, a seguir.

Figura 3 - Explicação sobre problemas combinatórios

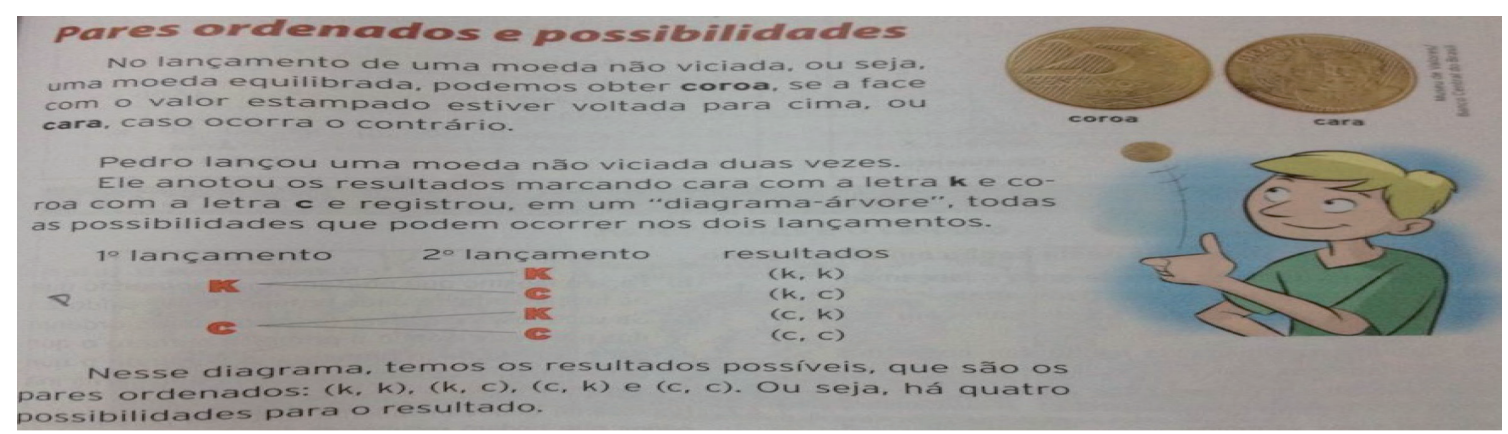

FONTE: Matemática - Ideias e Desafios. Iracema e Dulce. Saraiva Livr. Editores, 2012, 7o ano, p. 223.

Em linhas gerais, as questões estão em capítulos do bloco de Tratamento da Informação e envolvem a ideia de Probabilidade. Esse aspecto se diferencia do que foi visto nos anos iniciais, em que os problemas eram relacionados a capítulos de estrutura multiplicativa. Observando os conteúdos que a coleção aborda, possi- 
bilidades e chances aparecem em todos os livros, mesmo não encontrando algo sistematizado no 8o ano.

Almeida e Ferreira (2009) chamam a atenção para se ter consciência de que a Combinatória não é apenas uma técnica de cálculo de Probabilidade, mas, sim, que o raciocínio combinatório é parte importante para o entendimento deste conceito, existindo uma relação intrínseca entre as duas. Os livros precisam não apenas usar a Combinatória como um "gancho" para se aprender Probabilidade, mas levar em consideração todos os aspectos para que o conceito combinatório seja desenvolvido de forma satisfatória.

No Quadro 3, a seguir, apresentamos como são distribuídos esses problemas por significados da Combinatória ao longo da coleção.

Quadro 3: Quantitativo de problemas combinatórios distribuídos em cada ano escolar

\begin{tabular}{|c|c|c|c|c|}
\hline \multirow{2}{*}{$\begin{array}{c}\text { Ano } \\
\text { escolar }\end{array}$} & \multicolumn{4}{|c|}{ Quantidade de problemas combinatórios por significado } \\
\cline { 2 - 5 } & $\begin{array}{c}\text { Produto } \\
\text { Cartesiano }\end{array}$ & Combinação & Arranjo & Permutação \\
\hline $\mathbf{6}^{\mathbf{0}}$ ano & 9 & 10 & 5 & 6 \\
\hline $\mathbf{6}$ & 0 & 0 & 7 & 3 \\
\hline $\mathbf{8}^{\mathbf{0}}$ ano & 1 & 0 & 0 & 0 \\
\hline $\mathbf{9}^{\mathbf{0}}$ ano & 1 & 4 & 0 & 8 \\
\hline Total & $\mathbf{1 1}$ & $\mathbf{1 4}$ & $\mathbf{1 2}$ & $\mathbf{1 7}$ \\
\hline
\end{tabular}

Ao longo da coleção, os problemas de permutação apareceram com maior frequência, atingindo um percentual de 31,4\% de problemas. Em seguida, aparecem os de combinação com 25,9\% de problemas, seguidos dos de arranjo com 22,2\%. Os problemas de produto cartesiano apresentam o menor número, com 20,3\% de problemas. Essa distribuição dos tipos de problemas difere da apresentada nos anos iniciais, uma vez que, naqueles livros, os do tipo produto cartesiano aparecem com maior frequência e de permutação com menor frequência.

A diferença pode ser justificada pelo fato de os problemas de produto cartesiano serem de menor complexidade para crianças, e os de permutação de maior dificuldade para os alunos dos anos finais, os quais teriam maior maturidade intelectual para lidar com um maior número desses problemas. Percebemos também que houve um maior quantitativo de problemas nos livros dos anos finais, em comparação com o encontrado nos anos inicias, com um aumento de 42,1\%. Com relação aos significados da Combinatória presentes na coleção, pudemos notar que, no geral, todos são apresentados, porém por ano escolar isso não é constatado.

No que diz respeito às representações simbólicas apresentadas nas questões que envolvem o raciocínio combinatório, a coleção analisada deste ciclo de escolarização 
evidencia que os problemas, em sua maioria, apresentam um desenho junto com os enunciados $(55,5 \%)$ ou apenas o enunciado verbal da questão (33,3\%). Apenas quatro problemas $(7,4 \%)$ trazem árvores de possibilidades para ilustrar as situações, e 2 problemas $(3,7 \%)$ tabela/ quadro.

O uso de representações simbólicas junto com enunciados das atividades é um elemento importante que pode servir como apoio ao pensamento do aluno e auxiliar na resolução dos problemas. Entretanto, em sua maioria, é incluída, para além do enunciado verbal, uma figura que diz respeito ao contexto e não como sendo uma possibilidade de auxílio à resolução.

Com relação às representações simbólicas sugeridas para a resolução dos problemas, o Quadro 4 apresenta como essas sugestões estão distribuídas ao longo dos livros desta coleção.

Quadro 4. Frequência de representações simbólicas sugeridas nos problemas em cada ano escolar

\begin{tabular}{|c|c|c|c|c|c|c|}
\hline \multirow{2}{*}{$\begin{array}{c}\text { Ano } \\
\text { Escolar }\end{array}$} & \multicolumn{6}{|c|}{$\begin{array}{c}\text { Tipos de representações simbólicas sugeridas para a resolução das } \\
\text { atividades }\end{array}$} \\
\hline & $\begin{array}{l}\text { Apenas } \\
\text { enunciado }\end{array}$ & Algoritmo & Listagem & $\begin{array}{c}\text { Árvore de } \\
\text { possibilidades }\end{array}$ & $\begin{array}{l}\text { Tabela/ } \\
\text { Quadro }\end{array}$ & Outra \\
\hline $6^{0}$ & 14 & 0 & 1 & 4 & 11 & 0 \\
\hline $7^{0}$ & 8 & 0 & 0 & 1 & 0 & 1 \\
\hline $8^{\circ}$ & 1 & 0 & 0 & 0 & 0 & 0 \\
\hline $9^{\circ}$ & 8 & 1 & 0 & 4 & 0 & 0 \\
\hline Total & 31 & 1 & 1 & 9 & 11 & 1 \\
\hline
\end{tabular}

Esses dados permitem concluir que esta coleção, em mais da metade do quantitativo geral de problemas encontrados $(57,4 \%)$, não sugere qualquer representação para o aluno resolver as atividades, ou seja, fica ao critério do aluno escolher a melhor forma de solucionar as questões. Embora tenhamos encontrado outras formas de representação como a tabela/quadro (20,3\%), árvore de possibilidades (16,6\%), algoritmos e listagem (1\% cada), elas aparecem em menor frequência. Todavia, essas representações já apresentam uma indicação de como o aluno pode pensar sobre o problema, mesmo que de uma maneira menos formal que o uso de fórmulas, por exemplo.

No manual do professor não foi encontrada nenhuma orientação quanto aos significados da Combinatória, nem se fez menção aos invariantes de cada tipo de problema para que o professor possa trabalhá-los com os alunos. Essa ausência pode dificultar o trabalho do professor, uma vez que o livro didático é considerado um dos recursos mais utilizados em sala de aula e um importante orientador do trabalho docente. Ainda assim, há em dois volumes da coleção (6o e 9a ano), uma orientação 
para o professor quanto às estratégias de resolução, apresentando formas diferentes de resolver um mesmo problema.

Acreditamos que esse tipo de orientação deve estar presente em todos os livros escolares de Combinatória, uma vez que se faz importante o professor levar ao aluno diferentes formas de representar a solução de um problema para que ele encontre a mais adequada de acordo com a situação e com a sua forma de resolução.

Percebemos que os contextos envolvidos nos problemas desse ciclo de escolarização são mais diversificados, abrangendo, além das situações encontradas nos anos inicias, como jogo de dados, combinações de roupas e formação de números, também jogadas de moedas, pares de retas e medidas, que se inter-relacionam com outros conteúdos a serem estudados.

As variáveis continuam a tomar valores baixos, no que diz respeito ao número de possibilidades. A gradação de dificuldade relativa aos conteúdos em que a Combinatória intervém é percebida com relação aos problemas dos anos iniciais para os anos finais do Ensino Fundamental, relacionada aos contextos que envolvem o conhecimento de outros conteúdos, como tipos de retas, por exemplo.

\section{Considerações finais}

Analisando os dados, verificamos que os problemas combinatórios se fazem presentes nas coleções de livros didáticos analisadas do Ensino Fundamental, e há um aumento expressivo no quantitativo dessas questões dos anos iniciais para os anos finais. Tais problemas apresentam diferentes formas de abordagem dependentes do ciclo do Ensino Fundamental. Na coleção dos anos iniciais, eles aparecem relacionados às "estruturas multiplicativas" sem que haja um aprofundamento, mas, apenas, em exercícios a resolver. Já na coleção dos anos finais do Ensino Fundamental, tais problemas se incluem, de modo geral, em um capítulo específico no bloco Tratamento da Informação, envolvendo a ideia de Probabilidade, com explicações e exemplos.

Foram encontrados os quatro significados da Combinatória (produto cartesiano, arranjo, combinação e permutação) ao longo das coleções. Nos anos iniciais, existe uma predominância de questões de produto cartesiano e quase a inexistência de questões de arranjos. Nos anos finais, há uma melhor distribuição dos tipos de problemas de modo geral, importante para a compreensão plena da Combinatória, com maior quantitativo de questões de permutação e menor de produto cartesiano.

Na análise por ano escolar em todo o período do Ensino Fundamental, apenas encontramos o trabalho com os quatro significados da Combinatória num mesmo livro no 6o ano do Ensino Fundamental, enquanto nos demais são apresentados apenas alguns deles. Vergnaud (1986) defende que, para que o aluno compreen- 
da todas as propriedades de um campo conceitual, é necessário assumir que uma parte não representativa dessa diversidade não capta todas as propriedades de um conceito. Sendo assim, de modo geral, isso não é encontrado nos livros, segundo o ano escolar.

Com relação às representações simbólicas apresentadas nos problemas combinatórios, ainda que ocorra um alto índice de problemas apresentados apenas com o enunciado verbal, observamos que o desenho se destaca também como representação utilizada na abordagem das atividades propostas nas coleções analisadas no Ensino Fundamental, sendo, portanto, uma fonte de apoio ao pensamento dos alunos. No que diz respeito às sugestões apresentadas nas atividades de Combinatória, vimos variadas formas, tais como tabela, árvores de possibilidades e algoritmo. Entretanto, percebemos que, em ambos os ciclos, ainda são pouco frequentes essas indicações, mesmo sabendo-se das inúmeras formas do aluno pensar e solucionar um problema.

Na apresentação das atividades, ao longo das coleções analisadas no Ensino Fundamental, não foi possível perceber, na apresentação das atividades, uma proposta de percepção das propriedades invariantes envolvidas nos problemas combinatórios. De modo geral, as coleções analisadas não apresentam, no manual do professor, uma orientação específica para o ensino de Combinatória, sobre os diferentes tipos de problemas e suas particularidades. Ou seja, não é apresentada qualquer orientação quanto aos invariantes do conceito, aspecto importante para que o professor os trabalhe com os alunos e percepcione as lógicas implícitas em cada tipo de problema.

Os contextos dos problemas são adequados às faixas etárias dos alunos, embora nos anos iniciais sejam repetitivos para cada tipo de significado, e nos anos finais haja uma maior variedade. Foi possível observar uma gradação da dificuldade na abordagem dos problemas combinatórios na passagem dos anos iniciais para os anos finais do Ensino Fundamental. Essa gradação é dada a partir dos contextos que, no final deste nível de ensino, abrange outros conhecimentos, tais como Geometria.

Em resumo, foi possível apreender que, nas coleções de livros didáticos analisados destinados ao Ensino Fundamental, embora os problemas combinatórios estejam presentes e ocorra uma boa variedade de representações simbólicas, há de se abordar uma proposta de percepção das propriedades invariantes envolvidas neste campo conceitual e uma orientação específica para que os professores trabalhem tal conhecimento de forma a propiciar ao aluno a construção e o desenvolvimento desse conceito, principalmente no caso dos professores dos anos iniciais, para os quais, de um modo geral, não existe uma formação aprofundada da Combinatória. 


\section{Referências}

Almeida, A. L. de, \& Ferreira, A. C. (2009). Aprendendo análise combinatória através da resolução de problemas: um estudo com classes de 9o ano do Ensino Fundamental e 2o ano do Ensino Médio. Anais do 4o Encontro de Educação Matemática de Ouro Preto, Ouro Preto, MG, Brasil.

Assis, A., \& Magalhães, S. (2012). Amor, Roma, Mora: o raciocínio combinatório nos livros didáticos do 2o ao 5o ano do ensino fundamental. Anais do $4^{\circ}$ Encontro de Pesquisa Educacional de Pernambuco, Caruaru, PE, Brasil.

Barreto, F., Amaral, F., \& Borba, R. (2007). Como o raciocínio combinatório tem sido apresentado em livros didáticos de séries iniciais. TCC em Educação, Universidade Federal de Pernambuco, Recife.

Borba, R. (2013). Vamos combinar, arranjar e permutar: aprendendo combinatória desde os anos iniciais de escolarização. Anais do 11 oncontro Nacional de Educação Matemática, Curitiba, PR, Brasil.

Dante, L. R. (1996, janeiro/março). Livro didático de matemática: uso ou abuso? Em Aberto, ano $16,83-86$.

Fundação Nacional de Desenvolvimento da Educação (2013). Edital PNLD 2013. Retirado em 20 de janeiro de 2015, de : http://www.fnde.gov.br.

Merayo, F. (2001). Matemática discreta. Madri: Thomson Paraninfo S.A.

Mori, I., \& Onaga, D. (2012). Matemática - Ideias e Desafios (17a ed.). São Paulo: Saraiva Livretos Editores.

Ministério da Educação e Cultura. Secretaria de Ensino Fundamental. (1996). Guia de livros didáticos: PNLD 1997. 1a a 4aㅗ série. Brasília: autor.

Ministério da Educação e Cultura. Secretaria de Ensino Fundamental. (1997). Parâmetros Curriculares Nacionais. Matemática. 1o e 2o ciclos. Brasília: autor.

Ministério da Educação e Cultura. Secretaria de Ensino Fundamental. (1998). Parâmetros Curriculares Nacionais. Matemática. 3o e 4o ciclos. Brasília: autor.

Morgado, A., Pitombeira de Carvalho, J., Carvalho, P. \& Fernandez, P. (1991). Análise combinatória e probabilidade. Rio de Janeiro: Graftex.

Nunes, T., Campos, T., Magina, S., \& Bryant, P. (2001) Introdução à Educação Matemática: os números e as operações numéricas. São Paulo: PROEM.

Oliveira, E., \& Coutinho, C. (2013). Combinatória nos livros didáticos de matemática dos anos iniciais do ensino fundamental: uma análise do PNLD 2013. Anais do $11^{\circ}$ Encontro Nacional de Educação Matemática. Curitiba, PR, Brasil.

Padovan, D., Guerra, I. Milan, I., \& Monteiro, P. (2011). Projeto Prosa (2a ed.). São Paulo: Saraiva.

Pessoa, C. (2009). Quem dança com quem: o desenvolvimento do raciocínio combinatório do 2º ano do Ensino Fundamental ao 3a ano do Ensino Médio. Tese de Doutorado em Educação, Universidade Federal de Pernambuco, Recife.

Pessoa, C. (2014). O ensino de combinatória no ciclo de alfabetização. In C. R. Vianna, \& E. Rolkouski (Orgs.), Pacto nacional pela alfabetização na idade certa: Educação Estatística 
(pp. 39-50, v.1, la ed.). Brasília: Ministério da Educação, Secretaria de Educação Básica, Diretoria de Apoio à Gestão Educacional.

Pessoa, C., \& Borba, R. (2009). Quem dança com quem: o desenvolvimento do raciocínio combinatório de crianças de 1a a 4a série. Zetetiké, 17(39), 105-150.

Pessoa, C., \& Borba, R. (2010). O desenvolvimento do raciocínio combinatório na escolarização básica. Em Teia: Revista de Educação Matemática e Tecnológica Iberoamericana, 1(1), 1-22. Retirado em 10 de agosto de 2013, de http://emteia.gente.eti.br/index.php/emteia/ article/view/4

Rosa, H. (2004). Tópicos em resolução de problemas de combinatória. Anais do 8o Encontro Nacional de Educação Matemática. Recife, PE, Brasil.

Silva, P. (2014). Análise de livros didáticos do Ensino Médio acerca da forma de como são abordados os Problemas Combinatórios Condicionais. Anais do 18oㅡㄹ Entro Nacional de Estudantes de Pós-Graduação em Educação Matemática. Recife, PE, Brasil.

Vergnaud, G. (1986). Psicologia do desenvolvimento cognitivo e didática das matemáticas. Um exemplo: as estruturas aditivas. Análise Psicológica, 1, 75-90.

Recebido em: 03/10/2015

Aprovado em: 10/12/2015 